\title{
A Fast Electrical Energy Measurement Device and Power Consumption Detection Method of Electric Vehicle Charger
}

\author{
Zhao Fuping ${ }^{1}$, Liu Rongmei ${ }^{1}$, He Bei ${ }^{1}$, Zheng $\mathrm{Ke}^{1}$, Hu Xiaorui ${ }^{1}$, Ji Jing ${ }^{1}$, Hui Yan ${ }^{2}$ \\ ${ }^{1}$ Chongqing Electric Power Research Institute, Chongqing 401123, China \\ ${ }^{2}$ State Key Laboratory of Power Transmission Equipment \& System Security and \\ New Technology, Chongqing University, Chongqing 400044,China \\ $593222181 @ q q . c o m$
}

\begin{abstract}
More and more electric vehicles have been used in China, and many charging station has been built in China. Due to the reactive power loss caused by charging devices, the method to measure the power consumed by charging device is critical for power company. Based on impact loads generated by electric vehicles to the grid in fast charging mode, this paper studies the energy measurement method of electric vehicle charging mode. Since each phase current of charging post imbalance, to prevent the sum of each phase current vector of charging posts over the protection current threshold and the charging post breaker misusing in power circuit, preventing leakage circuit breaker current imbalance system is designed. Finally, this paper studies the power consumption detection methods of electric vehicle charging posts. By comparing the fast Fourier transform and wavelet analysis algorithms, an electric energy measurement device of electric vehicle charging machine based on FFT and wavelet analysis are proposed. Combined with fast data processing functions of DSP, the device can quickly and accurately measures electric vehicles charging amounts under different charging modes.
\end{abstract}

Keywords: FFT, wavelet analysis, electric vehicle charging machine, electric energy measurement

\section{Introduction}

In recent years, with the rapid development of electric vehicles, improving national energy security, reducing emissions and promoting smart grid development is of great importance. Due to the scale development of electric vehicles, a lot of public charging facilities are required. To guarantee electric vehicle charging metering accuracy and fairness after large scale, the State and the State Grid Corporation is devoting to develop relevant standards to regulate the charging machines energy metering technology, but the current research of the metering accuracy for the specific form of charging machine load is the lack of depth.

Electric vehicle charging modes include fast charge mode and slow charge mode. In the fast charge mode, the charge machine will generate impact loads to power grid, solving accurate measuring issues cased by waveform distortion under impact loads, which has been a new requires for energy metering system under the new situation of grid development [1,2]. Currently available electronic energy meter[3] on the market use fast Fourier transform (FFT) to analyze the sampled voltage and current waveforms, to obtain each harmonic component number and size, then calculate the harmonic power and determine the power flow. But the impact property of charging machine is a challenge for the FFT algorithm adaptability. Fast Fourier transform is very effective to analyzes the whole harmonic, but for unsteady harmonics, inter-harmonics or transient component, the 
Fourier transform is difficult to draw the correct amplitude[4]. Wavelet analysis method has a great advantage of metering the time-varying signal or non-stationary signals power, and have an ability of characterizing local information in the time domain and frequency domain [5]. As the dynamic range of the electric vehicle charging mode is wide, a comprehensive measurement error of charging machine should be taken into consideration, which can be used to guide to design the energy meter sensors, analog channels and A/D, to improve the metering algorithm adaptability. Combined with the advantage of the fast Fourier transform (fast Fourier transform, FFT) on the steady-state harmonic analysis and the advantage of wavelet analysis on the volatility and rapidly changing harmonic analysis, this paper proposes a method which combines wavelet transform and Fourier transform to meter electric vehicle energy. First, based on the influence of the impact load on the grid, the theory of electrical energy metering of impact loads is studied. Then Fourier transform and wavelet transform method of combining energy metering. And based on this analysis method, charge metering device is designed for electric vehicle charging machine. Then combining Fourier transform and wavelet transform method to energy metering. And based on this analysis method, the amount of charge metering device is designed for electric vehicle charging machine. In this paper, preventing leakage breaker current unbalanced system has been designed, to make the vector sum of each phase current within the prescribed scope. In order to measure both of the power dissipations accurately, a power consumption detection system for alternating current charging posts based on virtual load testing is proposed.

\section{Charging Machine Load Energy Metering Model}

\subsection{Charging Machine Load Theory Analysis}

At present, the domestic electric vehicle charging methods have fast charging and slow charging, sub-charging, clusters charging, wireless charging, mobile charging and energy storage charging etc. Fast charging and slow charging is the main modes ${ }^{[6]}$. Slow charging mode uses small current constant voltage or constant current to charge, the charging current is about $15 \mathrm{~A}$. Its charger and installation costs is relatively low, you can take full advantage of the power trough periods for charging to reduce charging costs, improve charging efficiency and extend battery life. The main drawback is the charging time is too long, it is difficult to meet the urgent operational requirements.

Fast charging mode can provide short-term charging service for electric vehicle in a larger current during the parking 20min 2h. Generally charging current is 150 400 A. under the fast charge mode, the electric vehicle will generate impact load to the grid, and its impact on the system is mainly making the waveform distortion seriously and irregular. As power changes rapidly, it is likely to cause system voltage flicker to make voltage waveform appears depressed or prominent. So impact load can case a great deal of random measurement error for the electric energy meter. The same meter to the same impact load in different time, its power metering amount may be vary considerably.

\subsection{Charging Machine Load Energy Metering Model}

This paper studies the impact load problem on the grid generated by the charging machine. Therefore, this model is charging machine load energy metering model based on impact load. While measuring the charging machine electrical energy, assuming the instantaneous real power in the measured point $\mathrm{A}$ is

$$
\begin{aligned}
& P_{A}(t)=u_{A}(t) i_{A}(t) \\
& u_{A}(t)=u_{1}(t)+u_{r}(t)
\end{aligned}
$$




$$
i_{A}(t)=i_{1}(t)+i_{r}(t)
$$

Where, $u_{1}(t), i_{1}(t)$ respectively is the fundamental voltage and fundamental current. $u_{1}(t), i_{1}(t)$ respectively is distortion voltage and distorted current contained in the electric vehicle charging machine.

Substitute equation (2) into equation (1), we have

$$
\begin{aligned}
P_{A}(t) & =\left[u_{1}(t)+u_{r}(t)\right] \times\left[i_{1}(t)+i_{r}(t)\right] \\
& =P_{1}(t)+P_{1 r}(t)+P_{r 1}(t)+P_{r}(t)
\end{aligned}
$$

The average power of Point $\mathrm{A}$ is

$$
P_{A}=P_{1}+P_{1 r}+P_{r 1}+P_{r}
$$

Where, $P_{1}$ is fundamental wave active power absorbed by load, $P_{1 r}$ is positive, indicating the active power generated by fundamental voltage and current, its value is zero when only containing the entire harmonic. $P_{\mathrm{r} 1}$ is negative, indicating the active power generated by distorted voltage and fundamental current, its value is zero when only containing the entire harmonic. $P_{1 r}$ and $P_{\mathrm{r} 1}$ are fed back to the grid in the way of the fundamental current, and do not pollute the grid. $P_{\mathrm{r}}$ is negative, indicating the active power generated by distorted voltage and distorted current, this part is fed back to the grid, and will cause pollution ${ }^{[7]}$.

According to the orthogonally of the trigonometric function, while there is only fundamental wave and integer harmonics in the grid, signal can be expressed as superposition of each sine waves.

Fundamental wave and each harmonic two-two pairwise orthogonal, each does not produce power. Namely, the value of $P_{\mathrm{r} 1}$ and $P_{1 r}$ in formula (5) is zero. Distortion power $P_{\mathrm{r}}$ is commonly referred as harmonic power $P_{h}$, then formula (5) can be simplified as

$$
P_{A}=P_{1}+P_{h}
$$

When the charging machine is charging, power systems generate impact load, distortion signal is not all sine wave, so orthogonal is absence, $P_{\mathrm{r} 1}$ and $P_{1 r}$ is not both zero ${ }^{[8]}$. Therefore, when metering the charging machine electrical energy, metering only fundamental wave power or metering only fundamental and harmonic power is unreasonable. $P_{\mathrm{r} 1}$ and $P_{1 r}$ can not be ignored in charging machine electrical energy metering. $P_{\mathrm{r}}$ is the energy from charging machine load back to grid. As it will be consumed by impedance in the grid lines, it should be removed from the energy measurement. Therefore, the electric vehicle charging machine active power reasonable measurement expression ${ }^{[7]}$ is

$$
\begin{aligned}
& P_{A}=P_{1}+P_{1 r}+P_{\mathrm{r} 1} \\
& \text { or can be simplified as } \\
& P=P_{A}-P_{\mathrm{r}}
\end{aligned}
$$




\section{Electric Vehicle Charging Energy Measurement Method Based on FFT and Wavelet Analysis}

\subsection{Harmonic Energy Metering based on FFT}

When the electric vehicle is fast charged by electric vehicle charging machine, it will cause grid voltage distortion to generate a large amount of harmonic, polluting the grid. Currently the method of harmonic measurement is mainly using Fourier analysis. In the power system, the non-sinusoidal waveform can usually be showed by the sum of fundamental wave and an unlimited number of higher harmonics, namely decomposing a function whose period is $\mathrm{T}$ into the form of the sum of innumerous trigonometric series ${ }^{[9]}$

$$
\begin{aligned}
f(t) & =a_{0}+\sum_{n=1}^{\infty} A_{n} \sin \left(n \omega_{1} t+\varphi\right)= \\
& =a_{0}+\sum_{n=1}^{\infty}\left(a_{n} \cos n \omega_{1} t+b_{n} \sin n \omega_{1} t\right)
\end{aligned}
$$

In the formula above: $a_{0}$ is DC component; $\omega_{1}$ is the fundamental angular frequency; $A_{n}$ and $\varphi$ are the amplitude and initial phase of the $n$-th harmonic; $a_{n}$ and $b_{n}$ are the cosine and sine coefficients of the $n$-th harmonic.

In order to analyze the actual harmonic energy waveforms of electric vehicle charging machine, it need to get the amplitude and phase of each harmonic by the exponential form of Fourier series. By Euler's formula (9) it derives the exponential form of Fourier series, as the following formula:

$$
\begin{aligned}
& f(t)=a_{0}+\sum_{n=1}^{\infty}\left(\frac{a_{n}-j b_{n}}{2} e^{j n w t}+\frac{a_{n}+j b_{n}}{2} e^{-j n w t}\right) \\
& =\left(\frac{a_{n}-j b_{n}}{2} e^{j n w t}\right)_{n=0}+ \\
& \sum_{n=1}^{\infty}\left(\frac{a_{n}-j b_{n}}{2} e^{j n w t}+\frac{a_{n}+j b_{n}}{2} e^{-j n w t}\right) \\
& =\sum_{n=-\infty}^{\infty} \frac{a_{n}-j b_{n}}{2} e^{j n w t}=\sum_{n=-\infty}^{\infty} \dot{F}_{n} e^{j n w t}
\end{aligned}
$$

Where:

$$
\begin{aligned}
& a_{0}=\left(a_{n} / 2\right)_{n=0}=\left(\frac{a_{n}-j b_{n}}{2} e^{j n w t}\right)_{n=0} \\
& \dot{F}_{n}=\frac{1}{2}\left(a_{n}-j b_{n}\right)=\frac{1}{2} \sqrt{a_{n}^{2}+b_{n}^{2}} e^{j \theta_{n}}=\frac{1}{2} A_{n} e^{j\left(\theta_{n}-90^{\circ}\right)} \\
& \theta_{n}=\operatorname{arctg}\left(-a_{n} / b_{n}\right)=\varphi_{n}-90^{\circ}
\end{aligned}
$$

When n $=0, \dot{F}_{n}=a_{0}$ is DC component.

By the formula (10) and (11) it can calculate the amplitude and phase of each harmonic voltage and current. Then it calculates the power and energy value for each harmonic and achieves the requirements of harmonic energy measurement.

\subsection{Harmonic Energy Metering based on Wavelet Analysis}

The wavelet transform is a wavelet function system which is composed by multi-scale refinement for time frequency domain ${ }^{[10]}$. In the distortion signal which is produced when 
electric vehicle is fast charged, it can use wavelet packet decomposition and reconstruction to process the signal. The principle of Wavelet Analysis electricity metering is to substitute the voltage and current signals into equation (1) in the sampling time $T$ (taken $n=2 \mathrm{~N}$ ), the active power of expression can be changed:

$$
\begin{aligned}
& P=\frac{1}{T} \int u(t) i(t) d t= \\
& \frac{1}{2^{N}} \sum_{i=0}^{2^{j}-1} \sum_{k=0}^{2^{N-j}-1} d_{u j}^{(i)}(k) d_{i j}^{(i)}(k)=\sum_{i=0}^{2^{j}-1} P_{j}^{(i)}
\end{aligned}
$$

In the formula above:

$$
P_{j}^{(i)}=\frac{1}{2^{N}} \sum_{k=0}^{2^{N-j}-1}\left[d_{u j}^{(i)}(k) d_{i j}^{(i)}(k)\right], \quad P_{j}^{(i)} \text { is the in-band signal power on the node } i
$$

when the decomposition scale is $j$, namely the active power of harmonic. Active energy with the sampling time can be expressed as:

$$
W=P T=\frac{1}{2^{N}} \sum_{k=0}^{2^{N-j}-1} P_{j}^{(i)} T=\sum_{k=0}^{2^{N-j}-1} W_{j}^{(i)}
$$

Where: $W_{j}^{(i)}=P_{j}^{(i)} T, W_{j}^{(i)}$ is the in-band signal energy on the node $i$ when the decomposition scale is $j$, namely the active energy of harmonics.

\subsection{Charging Machine Energy Metering based on FFT and Wavelet Analysis}

Fourier analysis method is a global transformation method and has the advantage of local analysis in the frequency domain and the disadvantage of no localization in the time domain. The wavelet analysis can well overcome the above disadvantage, but the Fourier transform method is better than the wavelet analysis method in the steady-state analysis.

In this paper, Fourier analysis and wavelet analysis are used to meter the electric energy of electric vehicle charging machine. When the electric vehicle is charging, it will produce an impact load in the grid and made the electromagnetic signal distortion. In this paper, the time of waveform distortion is determined by wavelet analysis, and then the harmonic wave is analyzed and calculated by Fourier analysis method and the wavelet analysis method. Specific signal processing is: the electric energy is calculated for every 4 cycles of the original signal. Firstly, using the wavelet transform method the signal is detected and distinguished for stationary signals, non-stationary signals and time-varying signal. Secondly, energy value of steady-state harmonic part point is measured by FFT and measuring unsteady wavelet analysis and partial energy values changed, energy values of non-stationary signals and time-varying are measured by the wavelet analysis method. Where, the measurement point begins from the previous cycle of mutation point. The energy measurement process is shown in Fig. 2.1. 


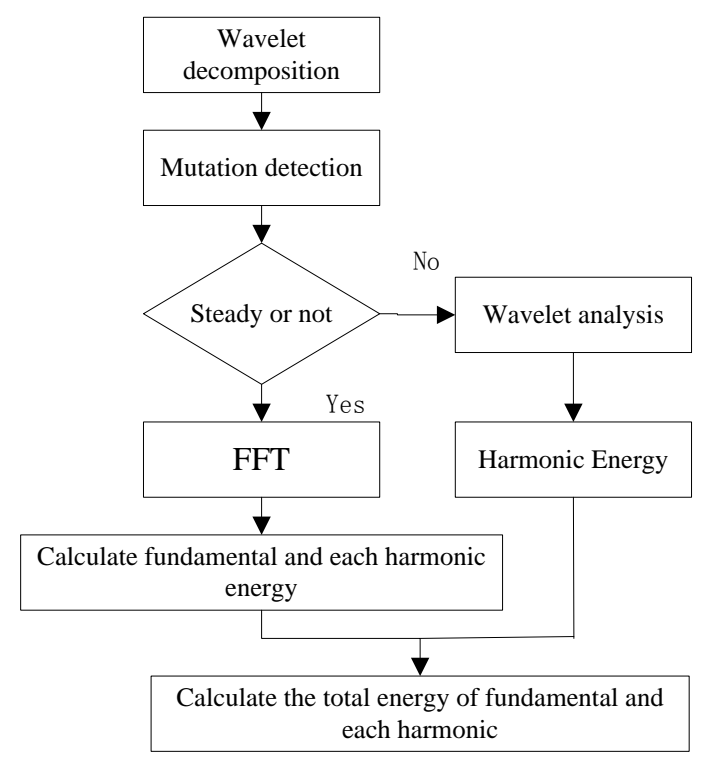

Figure 1. The Flow Chart of Energy Metering

\subsection{Technology of Prevent RCCB Unbalanced Current}

The residual current circuit breaker (RCCB) can break current of circuit automatically, when leakage current of circuit exceeds the predetermined value. The electric vehicle charging power circuit is made up of many parts, including breakers, watt-hour meters. Conventional charging posts imaginary load method need to make the breaker all interlinked into independent current. But as a result of each phase current imbalance, that is, the vector sum of each phase current will exceed the threshold of the breaker leakage protection current, and then the breaker disconnect for electric leakage protection, Eventually make verification could not be. To solve this problem, We have designed to prevent leakage breaker current unbalanced system, It can makes the vector sum of each phase current within the prescribed scope, As shown in Figure 2.

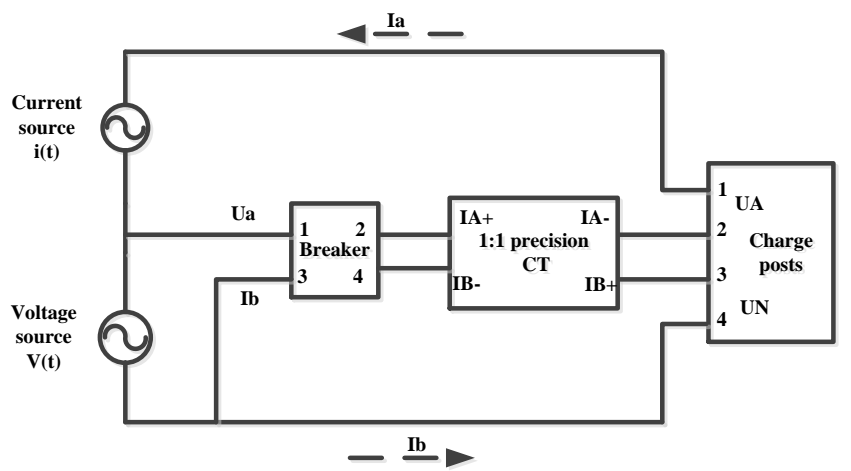

Figure 2. System Chart of Prevent RCCB Unbalanced Current

Its working principle is as follows:

Voltage source supply Ua in the figure, through the breaker of 1,3 pin input, By the 1:1 precision CT, Load to the UA current 2 feet and 4 feet of UN current of the Charging Point. Current Ia provided by the current source, pass through breaker 1 pin input, output 2 pin, By IA+、IA- of 1:1 precision CT, After charging posts UA 2 feet on the branch. Lastly, back to current source from 1 foot, which formed a current loop of the system. The 
secondary side current $\mathrm{Ib}$ of the 1:1 precision $\mathrm{CT}$, Output from the IB - through the breaker of 4 pin, 3 pin, UN current charging posts side of 4 pin and 3 pin in turn. Finally, flow back to the current transformer from the IB + feet, which formed another current loop of system.

In conventional virtual charging posts load test, by increasing the 1:1 precision CT to ensure the breaker of each phase current balance, which makes the error of each phase current vector sum within the prescribed scope. Depending on the size of the breaker rated current and leakage current protection, we can select corresponding grades of CT for fit.

\section{Power Consumption Detection Technology AND energy metering system Of Charger}

\subsection{Power Consumption Detection Technology of Charging Posts}

There will be huge inner power dissipation when charging posts works. The power consumption can be divided into static and dynamic power dissipation according to whether the charging posts is on battery charging state or not. The former is caused by voltage phase, the latter one owes to the complex effects current and voltage. To measure the power consumption of alternating charging posts precisely will help to charge customer fees more accurate and maintain the consumer benefits. In order to measure both of the power dissipations accurately, a power consumption detection system for alternating current charging posts based on virtual load testing is proposed in the paper and Fig. 4 gives the detailed structure illustration.

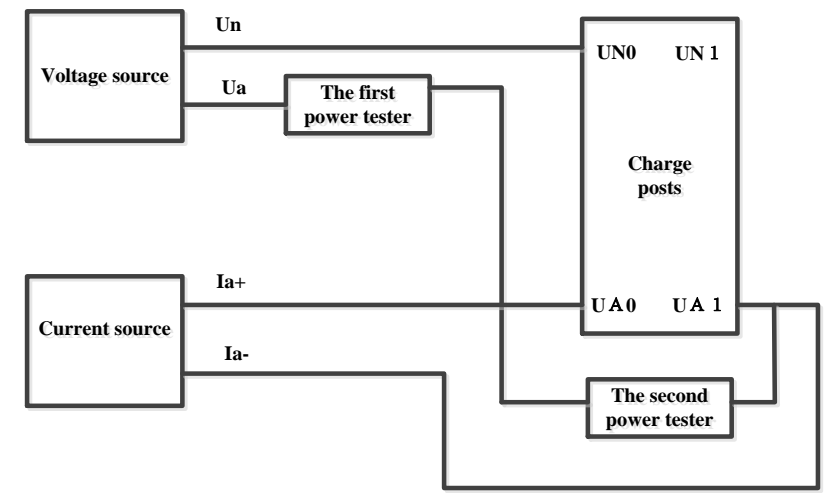

Figure 4. Scheme of Power Consumption Detection of Charging Posts

The detection process for static power dissipation:

1)Connect the voltage input port UNO of alternating current charging posts with the voltage output port Un of voltage source; then, connect the voltage output port Ua of voltage source with the voltage input port $\mathrm{Ua} 0$ of alternating current charging posts, and the first power consumption tester is in series with voltage input port UA0 and the voltage output port Ua.

2) Turn on the voltage source, the static value can be achieved immediately through the first power consumption tester.

The detection process for dynamic power dissipation:

1)Connect the voltage input port UNO of alternating current charging posts with the voltage output port Un of voltage source; then, connect the voltage output port Ua of voltage source with the voltage input port $\mathrm{Ua} 0$ of alternating current charging posts, and the first power consumption tester is in series with voltage input port UA0 and the voltage output port Ua.

2) Connect the current input port UA0 of alternating current charging posts with the voltage output port Ia+ of current source; then, connect the current output port Ia- of voltage source with the voltage input port UA1 of alternating current charging posts, and 
the second power consumption tester is in parallel with the voltage output port UA1 and the voltage input port UA0.

3) Turn on both the voltage source and current source, the power dissipation caused by voltage phase can be achieved immediately from the first power consumption tester, the power dissipation caused by current phase is obtained immediately from the second power consumption tester, the dynamic value is equal to sum of the two values.

\subsection{Design of Charger Energy Metering Device}

For the electric vehicle the key design point of the battery charger energy measurement device is to avoid distortion of the signal due to the impulse load when quickly charged with characteristics of wide bandwidth and large variations. Since only the same harmonic voltage and current component can reduce harmonic active, active power containing each harmonic component can be calculated as long as each harmonic voltage and current component is detected.

The measurement device firstly forms a discrete sequence with each phase current and voltage signal collected by acquisition circuit and then converts the sequence to the value of harmonic component by means of Fourier transform or wavelet packet analysis. Finally electric energy of each phase harmonic component can be calculated based on the method presented in the paper. Reasonable setting of the collection circuit plays an important role in the Accuracy of the electric energy estimation since the highest harmonic electric energy is decided by the sampling points of the program. Based on the principle above the hardware system of metering device for electric vehicles charger is designed utilizing the features of DSP and MAX125 FFT and wavelet analysis ${ }^{[11-14]}$.

The structure diagram of impulse load measurement is shown in Fig. 5. The system is divided into four parts including front-end signal disposal, sampling, electricity metering and control units.

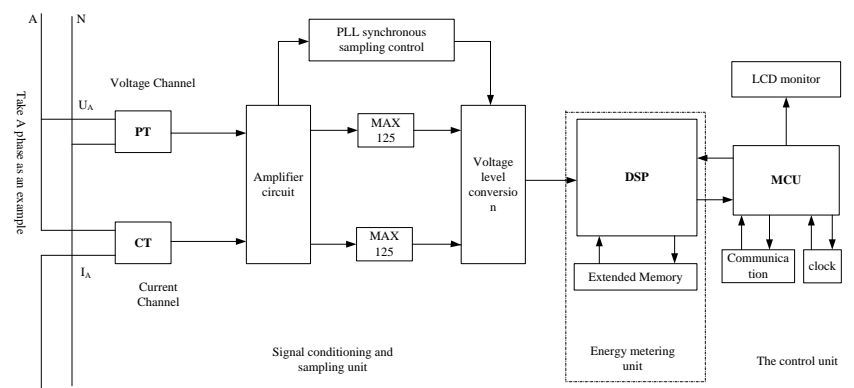

Figure 5. Charger Energy Metering System

PT, CT and amplifying circuit are the front-end signal disposal units which are used to convert the measured voltage and current signals to $-5 \mathrm{~V} \sim+5 \mathrm{~V}$ AC voltage signals proportionally. MAX125 serves as the core part of acquisition circuit which shifts the measured continuous analog signal to discrete digital signal. DSP circuit is the core part of Electricity metering unit which analyzes the sample data to calculate the charging power and electricity of charger. The proposed algorithm can be achieved because of the digital signal processing advantage of DSP chip. Control unit coordinates each unit of the system and completes other required functions with LCD monitor, clock chip and communication interface.

\section{Conclusion}

Along with the rapid development of electric vehicles and mature electronic technology fair and reasonable measurement of electric vehicle charging capacity is being more and more important. Combing the advantages of FFT for steady-state harmonic analysis and wavelet analysis for distortion signal processing this paper designs a charging metering 
device for electric vehicle upon the union of FFT and wavelet analysis. Current unbalanced prevention system of residual current circuit-breaker and charging posts power consumption detection system are designed, based on these modules, an electric vehicle charging posts(charger) detection method and device is proposed. The charging metering device in this paper adopts AC source isolated sampler, A/D sampler and so on to obtain charging waveform signal. It can quickly calculate the charging power of electric car charger with the computing method propose in the paper and ability to deal with the data quickly of DSP. It supplies technology leader to exploit energy meter with more accuracy to calculate the charging power due to the feasibility to impulse load.

\section{References}

[1] Tan Zhiqiang, Peng Chuning. The influence on electric energy measurement under the condition of impulse load [J]. Electrical Measurement \& Instrumentation, 2003, 40(1): 41 -43.

[2] J.Wang, K.-H.Wu and Z.-Z.Liu. "The influence of the electric vehicle charging for distribution network load and orderly control research,"(in Chinese)Electric Power Automation Equipment,Vol.33,No.8, pp.47-52,China,2013.

[3] Liu Wensong, Liu Shaohua, Wang Yunjing. Harmonic electric energy measuring technique. [J]. China Electric Power Education, 2010(35): 255-257.

[4] R.Zhao. "The design and research of EVs AC charging spot,"M.S. thesis, Dept. Mechatron. Eng., Soochow University., Suzhou ,China,2013.

[5] Rui Guosheng, Kang Jian. The wavelet and Fourier analysis. [M]. Bei Jing: Electronic Industry Press, 2010: $39-57$.

[6] Zhang Tao. Investigation of electric energy measuring problems regarding charge of electric vehicles. [J]. China Urban Economy, 2011, 26: 145.

[7] Zhang Xiaobing. Electrical network power flow analysis and research on new measuring methods of electric energy on the condition of distorted signals. [D]. Harbin: Harbin University of Science and Technology, 2007.

[8] Yue Liangjing, Xu Yong, Xiong Wenqing. Design on electric energy meter of impact load based on lifting wavelet transforms [J] Transducer and Microsystem Technologies, 2012, 31(5): 110-114.

[9] Cao Huimin. Harmonic affection on power measurement installation. [D]. Baoding: North China Electric Power University, 2010.

[10] Zhang Bao, Yi Jiliang, Jiangjianwei. [D]. Journal of Hunan University of Technology, 2013, 27(5): 58-62.

[11] X-S.Liu."Research on the design and control method of electric vehicle charging posts, "M.S. thesis, Dept. Control theory and control. Eng.,GuangXi GuangXi University Of Science And Technology., China, 2013.

[12] H. Pang, D. Li, Y. Zu,"An improved algorithm for harmonic analysis of power system using FFT technique". Proceedings of the Csee, vol. 6(009). 2003

[13] Zhang Bao, Yi Jiliang, Jiangjianwei. [D]. Journal of Hunan University of Technology, 2013, 27(5): 58-62.

[14] Jin Shouxing. Development of a device for metering harmonic energy in electric power system based on DSP. [D]. Dalian: Dalian University of Technology, 2005.

\section{Authors}

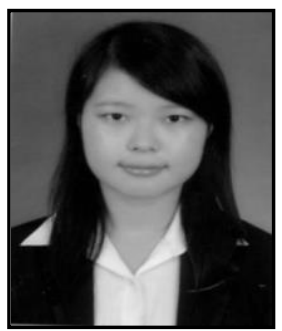

\section{Hui Yan}

Born in 1991,pursuing her detection method of electric vehicle charger researches. 
International Journal of Grid and Distributed Computing Vol. 9, No. 9 (2016) 